\title{
Congenital cerebral malformations and dysfunction in fetuses and newborns following the 2013 to 2014 Zika virus epidemic in French Polynesia
}

\author{
M Besnard ${ }^{1}$, D Eyrolle-Guignot ${ }^{2}$, P Guillemette-Artur $^{3}$, S Lastère ${ }^{4}$, F Bost-Bezeaud $^{5}$, L Marcelis $^{6}$, V Abadie $^{7}$, C Garel $^{8}$, M \\ Moutard 9 , J Jouannic ${ }^{10}$, F Rozenberg ${ }^{112}{ }^{12}$, I Leparc-Goffart ${ }^{12}{ }^{13}$, H Mallet $^{6}$ \\ 1. Service de réanimation néonatale, Centre Hospitalier de Polynésie Française (CHPF), Pirae, Polynésie Française \\ 2. Service d'obstétrique, CHPF, Pirae, Polynésie Française \\ 3. Service de radiologie, CHPF, Pirae, Polynésie Française \\ 4. Laboratoire de biologie médicale, CHPF, Pirae, Polynésie Française \\ 5. Laboratoire d'anatomie pathologique, CHPF, Pirae, Polynésie Française \\ 6. Bureau de Veille Sanitaire, Direction de la Santé, Papeete, Polynésie Française \\ 7. Service de pédiatrie générale, Assistance Publique-Hôpitaux de Paris, (AP-HP), Hôpital Necker, Paris, France \\ 8. Service de radiologie, AP-HP, Hôpital Armand-Trousseau, Paris, France \\ 9. Service de neuro-pédiatrie, AP-HP, Hôpital Armand-Trousseau, Paris, France \\ 10. Service de Médecine Fœtale, AP-HP, Hôpital Armand-Trousseau, Paris, France \\ 11. Service de virologie, AP-HP, Hôpital Cochin, Paris, France \\ 12. These authors contributed equally to the manuscript \\ 13. Centre National de Référence des arbovirus, IRBA, Marseille, France
}

Correspondence: Marianne Besnard (mbesnard@nohao.net)

Citation style for this article:

Besnard M, Eyrolle-Guignot D, Guillemette-Artur P, Lastère S, Bost-Bezeaud F, Marcelis L, Abadie V, Garel C, Moutard M, Jouannic J, Rozenberg F, Leparc-Goffart I, Mallet H. Congenital cerebral malformations and dysfunction in fetuses and newborns following the 2013 to 2014 Zika virus epidemic in French Polynesia. Euro Surveill. 2016;21(13):pii=30181. DOI: http://dx.doi.org/10.2807/1560-7917.ES.2016.21.13.30181

We detected an unusual increase in congenital cerebral malformations and dysfunction in fetuses and newborns in French Polynesia, following an epidemic of Zika virus (ZIKV), from October 2013 to March 2014. A retrospective review identified 19 cases, including eight with major brain lesions and severe microcephaly, six with severe cerebral lesions without microcephaly and five with brainstem dysfunction without visible malformations. Imaging revealed profound neurological lesions (septal and callosal disruption, ventriculomegaly, abnormal neuronal migration, cerebellar hypoplasia, occipital pseudocysts, brain calcifications). Amniotic fluid was drawn from seven cases at gestation weeks 20 to 29. ZIKV RNA was detected by RT-PCR and infectious ZIKV isolates were obtained in four of five microcephalic, but not in two non-microcephalic cases with severe brain lesions. Medical termination of pregnancy was performed in eleven cases; two cases with brainstem dysfunction died in the first months of life; six cases are alive, with severe neurological impairment. The results show that four of seven tested fetuses with major neurological injuries were infected with ZIKV in utero. For other non-microcephalic, congenital abnormalities we were not able to prove or exclude ZIKV infection retrospectively. The unusual occurrence of brain malformations or dysfunction without microcephaly following a ZIKV outbreak needs further studies.

\section{Introduction}

A Zika virus (ZIKV) outbreak in French Polynesia from October 2013 to March 2014 resulted in 8,750 suspected cases reported through the general practitioners-based (25 to 45 sentinel practitioners) sentinel surveillance system for infectious diseases. The system exists since 2009 and syndromic cases definitions are basis for weekly reporting. An estimated 32,000 suspected cases sought medical care and more than half of the population might have been infected $[1,2]$. ZIKV is an emerging arbovirus that before 2013 was considered to cause only mild disease, characterised by fever, rash, joint pain, and conjunctivitis.

From 2014 to 2015, following the ZIKV epidemic, we observed an unusual increase in annual congenital cerebral malformations (two-fold), brainstem dysfunction (31-fold), and severe microcephaly (14-fold) among fetuses and newborns (data not shown). Following the announcement by the Brazilian Government in November 2015 of a dramatic increase in the incidence of microcephaly possibly associated with an ongoing ZIKV outbreak [3,4], we notified the World Health Organization (WHO) of this unusual cluster of congenital neurological abnormalities in our islands [5].

French Polynesia consists of five archipelagos in the South Pacific Ocean, with 118 islands, of which 76 are inhabited. The total population was 271,800 in 2014 


\section{FIGURE 1}

Prenatal MRI T2-weighted performed at 30 weeks plus 5 days of gestation on fetus with congenital cerebral malformations following the 2013-2014 Zika virus outbreak, French Polynesia, 2014 to 2015

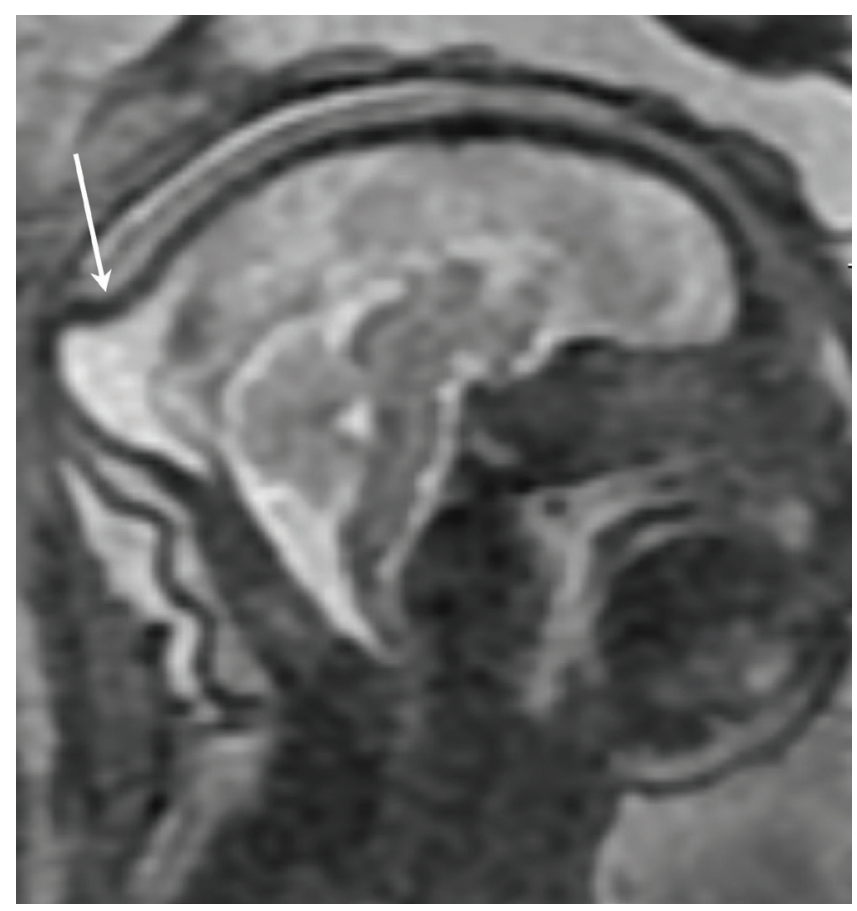

This midsagittal slice shows very small volume of supratentorial structures and the pericerebral space is enlarged. We see a notch over the fetal skull (arrow). The corpus callosum and the cavum septi pellucidi are absent.

and $70 \%$ reside on Tahiti. Approximately 4,200 births are recorded per year (2014: 4,161 births), mainly in Tahiti, with $60 \%$ at the Centre Hospitalier de Polynésie Française (CHPF), 30\% in two private clinics in Tahiti, and $10 \%$ in two distant district hospitals.

We report here a retrospective case series of 19 fetal and newborn cases with congenital cerebral malformations and dysfunction and detail the neurological lesions identified and the corresponding virological results.

\section{Methods}

We conducted a retrospective review of congenital cerebral malformations and dysfunction, detected in a prenatal and neonatal population in French Polynesia from March 2014 to May 2015. We included pregnancies beginning between June 2013 and August 2014, the period which corresponded to the largest estimated circulation period of ZIKV. There is no territorial register of congenital malformations, thus the prenatal cases were collected through the non-computerised charts of the prenatal diagnostic unit of the CHPF and the neonatal cases were recorded through the hospital Programme de Médicalisation des Systèmes d'Information (PMSI).

\section{FIGURE 2}

Prenatal MRI T2-weighted performed at 28 weeks plus 4 days of gestation on fetus with congenital cerebral malformations following the 2013-2014 Zika virus outbreak French Polynesia, 2014 to 2015

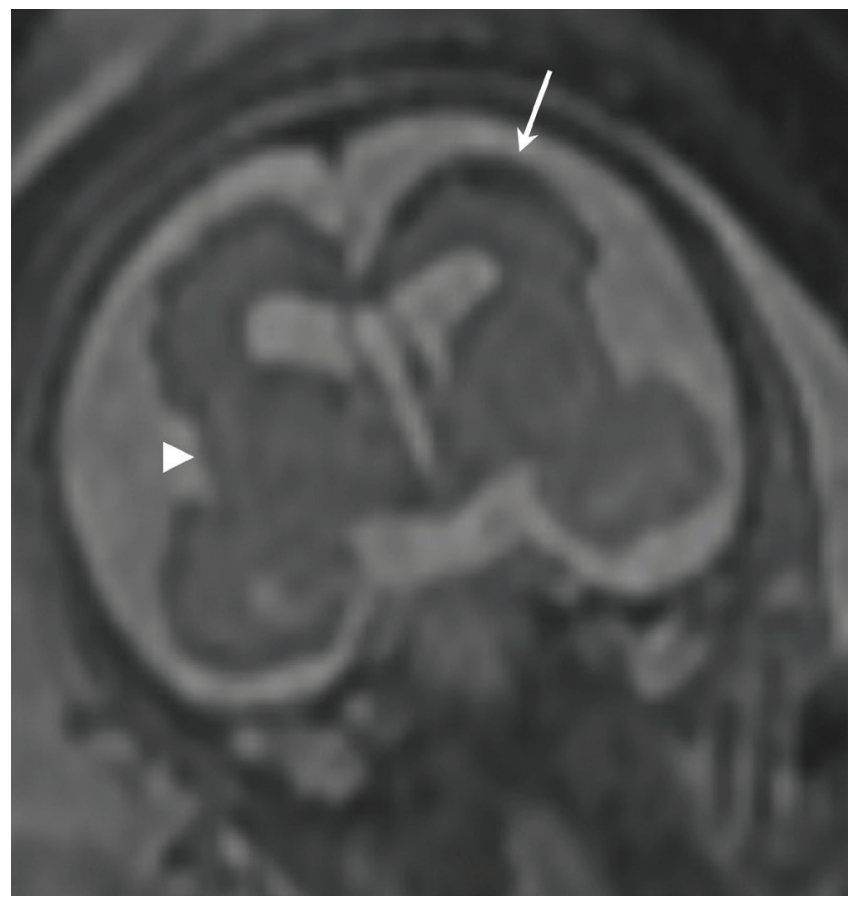

This coronal slice shows very irregular cortex (arrow), which is suggestive of diffuse polymicrogyria with bilateral opercular dysplasia (arrowhead) and enlarged pericerebral space. The corpus callosum and the cavum septi pellucidi are seen.

\section{Prenatal monitoring}

According to the recommendations of the Haute Autorité de Santé, France [6], three prenatal ultrasounds (US) scans, trisomy screening and serological assays for, hepatitis B, HIV, rubella, toxoplasmosis and syphilis are performed for all pregnant mothers. When fetal anomalies are detected by routine antenatal US scan, the pregnant woman is referred to the CHPF prenatal diagnostic unit, where a second US evaluation is conducted by an expert obstetrician and a nurse experienced in prenatal diagnosis. Once cerebral congenital malformations, for example, are confirmed, they perform an amniocentesis and prescribe magnetic resonance imaging $(\mathrm{MRI})$ of the fetal brain. Then, as all cases of severe congenital abnormalities, they are reviewed by the Prenatal Multidisciplinary Diagnostic Centre of East Paris, France. According to the French law, the committee determines if termination of pregnancy (TOP) can be performed, until full gestational term, upon parental request. An average of 20 annual TOPs are performed (range 12-26) for various severe fetal malformations that constitute a substantial risk of serious motor or cognitive disabilities. After the TOP procedure, pictures of the fetus are taken and skeletal X-rays are performed. Weight, height and head circumference are measured. No autopsy is done as there is no fetopathologist in French Polynesia. Except for 


\section{FIGURE 3}

Prenatal ultrasound (a) performed at 27 weeks of gestation and prenatal MRI T2-weighted (b) performed at 30 weeks plus 1 day of gestation on fetus with congenital cerebral malformations following the 2013-2014 Zika virus outbreak, French Polynesia, 2014 to 2015

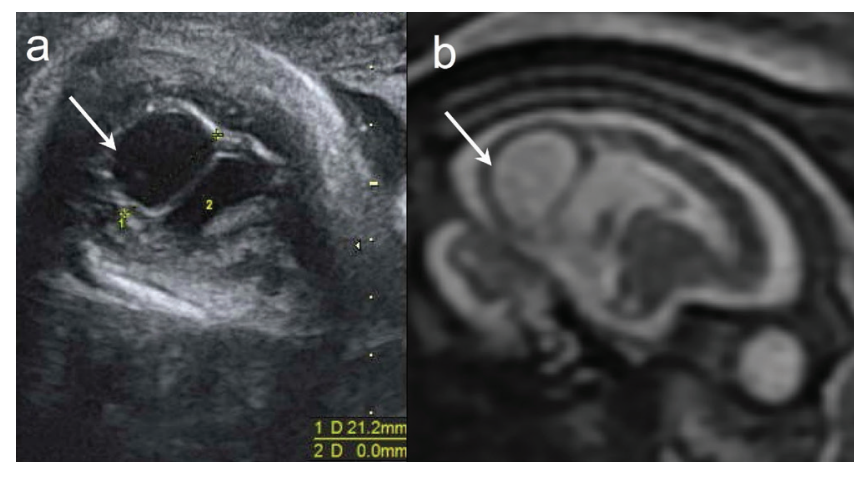

These para-sagittal slices show a large occipital subependymal pseudocyst (arrow) facing the enlarged occipital horn and pericerebral space is enlarged.

formalin-fixed, paraffin-embedded placenta samples, no fetal tissue is stored.

\section{Postnatal diagnosis}

There is only one neonatal intensive care unit (ICU) in French Polynesia. It is situated at CHPF and takes care of all severe neurological malformations and dysfunction. We collected all cases by reviewing the coding of hospitalised patients [7]. When congenital cerebral malformations are detected in utero, systematic control after birth of brain imaging (US, MRI or CT-scan) are performed to confirm and assess the brain lesions.

\section{Case definition}

We included cases with congenital microcephaly, defined by a head circumference below the third percentile for gestational age and sex, according to the Association des Utilisateurs de Dossiers Informatisés en Pédiatrie, Obstétrique et Gynécologie (AUDIPOG) charts [8], cases with brain lesions without microcephaly and cases with congenital brainstem dysfunction characterised by a deficiency of coordination between sucking, swallowing and breathing, with no visible cerebral malformation and a normal birth head circumference. We assigned them to three groups:

Group 1: fetuses (Group 1a) and newborns (Group 1b) with severe cerebral lesions and microcephaly;

Group 2: fetuses with severe cerebral lesions without microcephaly;

Group 3: newborns with congenital brainstem dysfunction.

Cases with proven aetiology and usual and isolated neurological anomalies without brain damage were excluded.
We retrospectively reviewed the medical records of all mothers and fetuses or newborns with their clinical, serological and radiological (US scans and MRI) data. The imaging examinations were reviewed by radiologists of CHPF and of Hospital Armand-Trousseau, Paris.

We contacted mothers of cases identified and asked about symptoms of ZIKV infection during their pregnancy. Informed written consent was obtained from mothers for all investigations regarding available samples, and publication. Our study was approved by the Ethics Committee of French Polynesia.

\section{Laboratory investigations}

Amniotic fluids were analysed for karyotype (comparative genomic hybridisation (CGH) array was not routinely performed) and PCR for cytomegalovirus (CMV). Between June 2013 and August 2014, no virological testing for ZIKV was performed in amniotic fluids and pregnant women.

Retrospectively, we collected seven of thirteen available amniotic fluids, drawn from 11 mothers with fetuses with congenital brain malformations $(5 / 5$ in Group 1a; $2 / 6$ in Group 2) and 2 mothers with fetuses with polyhydramnios (o/2 in Group 3), reflecting future brainstem dysfunction, and tested them by RT-PCR for dengue virus (DENV), enteroviruses (EV), herpes simplex virus (HSV), lymphocytic choriomeningitis virus (LCMV), rubella, and varicella zoster virus (VZV). ZIKV was tested with RT-PCR (RealStar ZIKV RT-PCR 1.0, Altona Diagnostics, Germany) and cultured on Vero cells and C6/36 cell lines. ZIKV serology was investigated with ELISA to detect flavivirus antibodies and seroneutralisation assays to further characterise the probable flavivirus.

\section{Results}

There were 4,787 births during the study period March 2014 to May 2015, and we observed 33 cases with congenital brain malformations or dysfunction. We excluded four cases with proven aetiology (one toxoplasmosis, two chromosomal abnormalities, one myopathy) and ten with usual and isolated neurological anomalies without brain damage (three intraventricular haemorrhages, three corpus callosum agenesis, one neural tube defect and three polymalformative syndromes during the first trimester of pregnancy). The remaining unusual 19 congenital cerebral malformations and dysfunction were included in our study: eight in Group 1 i.e. five fetuses in Group $1 \mathrm{a}$ and three newborns in Group 1b; six fetuses in Group 2 and five newborns in Group 3 (Table 1).

The mean age of the mothers in Group 1 was 29.7 years (range 22.8-38.9), 26.9 years in Group 2 (range 15.738.0), and 30.8 years in Group 3 (range 20.2-39.0). There was no relevant medical or family genetic history in any of the mothers. Alcohol or maternal cocaine use during pregnancy was denied in our series. 


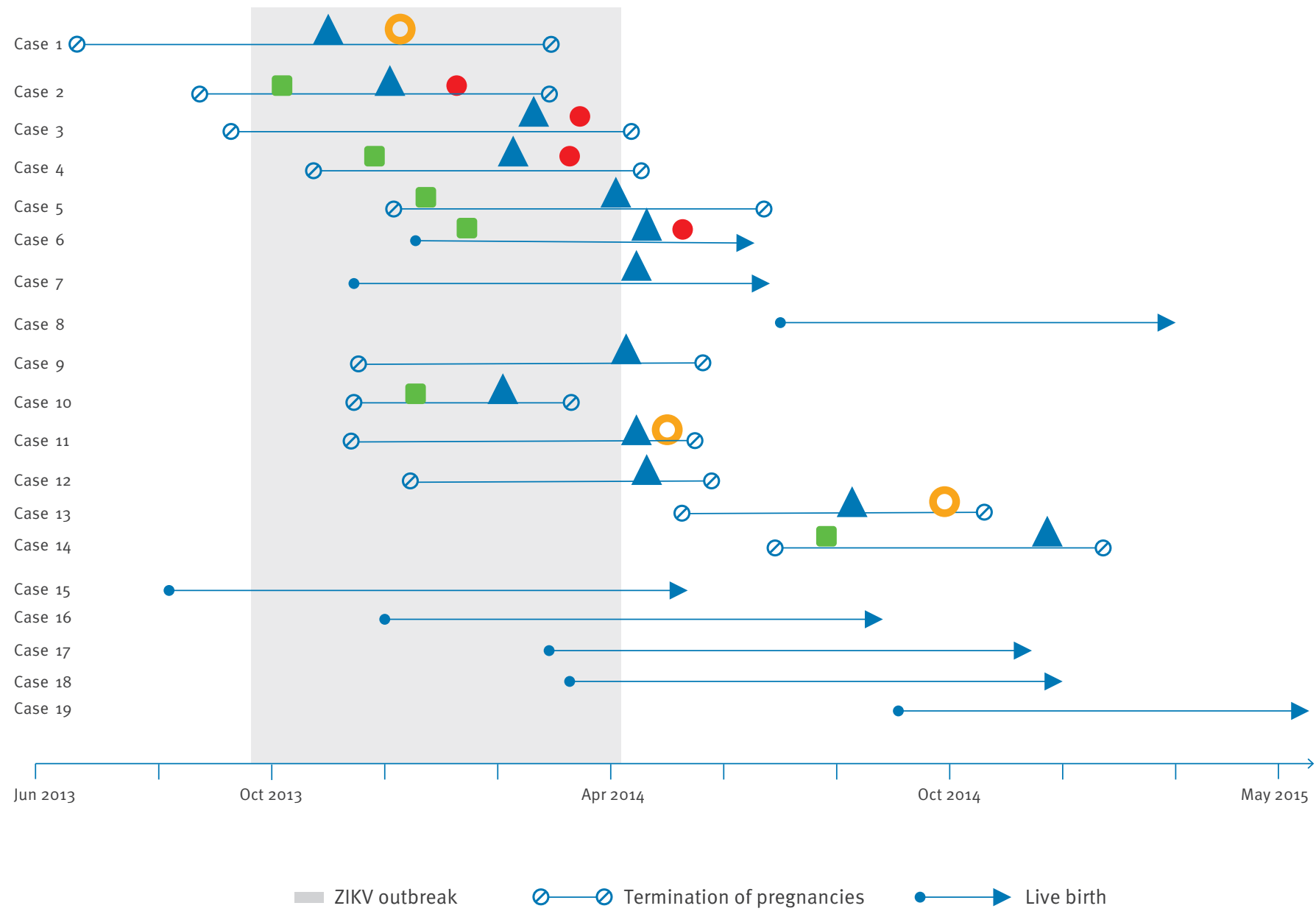

US scan showing brain abnormalities
Mother experiences symptoms of ZIKV infection
ZIKV RT-PCR positive

ZIKV RT-PCR negative

US: ultrasound; ZIKV: Zika virus.

Symbols for ZIKV PCR indicate the time when amniotic fluid samples were collected; testing was undertaken retrospectively in December 2015 .

Information about ZIKV infection symptoms in mothers was obtained retrospectively in December 2015.

There was no seroconversion for CMV, hepatitis B, HIV, rubella, syphilis and toxoplasmosis in any of the mothers; for 7 of 13 samples of amniotic fluid, PCR for EV, rubella, LCMV, HSV, VZV and DENV was negative.

Imaging findings for all cases are summarised in Table 2. Skeletal X-rays were done for all fetuses and revealed an intracranial calcification in one case (Case 5), which was also observed on prenatal US scan.

Below we describe in detail the findings in the different Groups.

\section{Group 1a}

The five fetuses in this group exhibited severe signs of brain injury associated with microcephaly, visualised within the second trimester of pregnancy (after 20 weeks of gestation (WG)) by US scan and confirmed by MRI in four cases. In all cases, in addition to microcephaly, imaging findings (fetal US or MRI) showed multiple severe cerebral injuries including profound destruction of median structures and evidence of interruption of brain development (Figures $1,2,3$ ) including absence (or rupture) of the corpus callosum $(n=3)$ and of cavum septi pellucidi $(n=3)$, ventriculomegaly superior or equal to $12 \mathrm{~mm}$ (norm: «10 mm, mild ventriculomegaly $10-12 \mathrm{~mm})(\mathrm{n}=3)$, occipital subependymal pseudocysts $(n=2)$, opercular dysplasia (agyria, 
Main characteristics of fetuses and newborns with congenital cerebral malformations and dysfunction following the 2013-2014 Zika virus outbreak, French Polynesia, 2014 to 2015 (n=19)

\begin{tabular}{|c|c|c|c|c|c|c|}
\hline & $\begin{array}{l}\text { Date of } \\
\text { conception }\end{array}$ & $\begin{array}{l}\text { Gestational term at birth or } \\
\text { TOPa } \text {, WG + D }\end{array}$ & $\begin{array}{l}\text { Head circumference at } \\
\text { birth, percentile }\end{array}$ & $\begin{array}{l}\text { RT-PCR ZIKV in } \\
\text { amniotic fluid }\end{array}$ & Karyotype & $\begin{array}{l}\text { Birth weight, } \\
\text { percentile }\end{array}$ \\
\hline \multicolumn{7}{|c|}{ Group 1a: Fetal brain abnormalities and microcephaly } \\
\hline Case 1 & 17 Jun 2013 & $39+4 a$ & $<3^{\circ}$ & Neg & $46 X Y$ & $10^{\circ}$ \\
\hline Case 2 & 15 Aug 2013 & $30+1 a$ & $<3^{\circ}$ & Pos & $46 \times X$ & $50^{\circ}$ \\
\hline Case 3 & 29 Sep 2013 & $31+4 a$ & $<3^{\circ}$ & Pos & $46 X Y$ & $75^{\circ}$ \\
\hline Case 4 & 22 Oct 2013 & $26+1 a$ & NA & Pos & $46 X Y$ & $75^{\circ}$ \\
\hline Case 5 & 20 Dec 2013 & $21+4 a$ & NA & Pos & $46 X Y$ & $5^{\circ}$ \\
\hline \multicolumn{7}{|c|}{ Group 1b: Newborns with cerebral lesions and severe microcephaly } \\
\hline Case 6 & 7 Oct 2013 & 41 & $<3^{\circ}$ & NA & NA & $4^{\circ}$ \\
\hline Case 7 & 10 Nov 2013 & $36+4$ & $<3^{\circ}$ & NA & NA & $11^{\circ}$ \\
\hline Case 8 & 22 Jul 2014 & $36+2$ & $<3^{\circ}$ & NA & $46 X Y$ & $32^{\circ}$ \\
\hline \multicolumn{7}{|c|}{ Group 2: Fetal brain abnormalities without microcephaly } \\
\hline Case 9 & 25 Oct 2013 & $24 a$ & NA & NA & $46 X Y$ & $10^{\circ}$ \\
\hline Case 10 & 5 Nov 2013 & $26+6 a$ & NA & NA & $46 \times x$ & $25^{\circ}$ \\
\hline Case 11 & 24 Nov 2013 & $29+3 a$ & $5^{\circ}$ & NA & $46 X Y$ & $63^{\circ}$ \\
\hline Case 12 & $18 \mathrm{Dec} 2013$ & $21+5 a$ & $5^{\circ}$ & $\mathrm{Neg}$ & $46 x x$ & $5^{\circ}$ \\
\hline Case 13 & 17 May 2014 & $23+5 a$ & $90^{\circ}$ & $\mathrm{Neg}$ & $46 X Y$ & $80^{\circ}$ \\
\hline Case 14 & 22 Aug 2014 & $26 a$ & $25^{\circ}$ & $\mathrm{NA}$ & $46 x x$ & $60^{\circ}$ \\
\hline \multicolumn{7}{|c|}{ Group 3: Newborns with congenital brainstem dysfunction } \\
\hline Case 15 & 5 Sep 2013 & $39+4$ & $45^{\circ}$ & NA & $46 \times X$ & $25^{\circ}$ \\
\hline Case 16 & 20 Dec 2013 & $39+3$ & $45^{\circ}$ & NA & $46 \times X$ & $4^{\circ}$ \\
\hline Case 17 & 18 Mar 2014 & $35+4$ & $75^{\circ}$ & NA & $46 \times X$ & $67^{\circ}$ \\
\hline Case 18 & 11 Mar 2014 & $40+1$ & $7^{\circ}$ & NA & $46 X Y$ & $15^{\circ}$ \\
\hline Case 19 & 7 Sep 2014 & 39 & $70^{\circ}$ & NA & $46 X Y$ & $51^{\circ}$ \\
\hline
\end{tabular}

D: day; NA: not available; Neg: negative; Pos: positive; TOP: termination of pregnancy; WG: weeks of gestation.

a Term $(W G+D)$ at TOP.

polymicrogyria) $(n=4)$, vermian dysgenesis $(n=3)$, enlarged pericerebral space $(n=4)$ and parenchymal calcifications $(n=5)$. In each of the five cases at least five of these lesions were present and all pregnancies were terminated. Extracranial findings were micropenis (Case 1 ) and hypotrophy below the third percentile with intestinal hyperechogenicity (Case 5). At delivery, head circumferences were below the third percentile, but not measured on two fetuses. Placental microcalcifications were observed in three cases either on US scan and histology (Case 3) or histologically only (Cases 4 and 5).

Retrospectively, ZIKV RNA was detected by RT-PCR and infectious ZIKV was isolated in four of five amniotic fluid samples (Cases 2, 3, 4, 5). When interviewed, the mothers of Cases 2, 4 and 5 reported clinical infection in the first trimester of pregnancy, the mother of Case 3 could not be reached. The chronological sequence of maternal symptoms, fetal malformations discovery and PCR results are summarised in Figure 4.

\section{Group $1 \mathrm{~b}$}

Microcephaly was diagnosed in utero for two of the three cases (Case 7 and 8) but mothers did not wish
TOP. Microcephaly was diagnosed for one case (Case 6) on US scan at 34 WG and the mother did not wish further investigation. MRI was performed in utero for one case (Case 7), and one month after birth in another (Case 8); in both cases MRI confirmed severe microcephaly and cerebral lesions with mainly, occipital pseudocysts and abnormal gyration (Table 2). All three cases were born with a head circumference of $27 \mathrm{~cm}$, which was substantially below the third percentile. At birth, one case was hypotrophic with birth weight at fourth percentile (Case 6) and one had micropenis and severe vision deficiency due to bilateral ocular atrophy and hearing loss suspected on potential recalls (Case 8). No cutaneous lesion or malformation were observed. All three cases demonstrated severe neurological outcome manifest by delayed motor and cognitive development, failure to thrive due to swallowing difficulties and epilepsy.

Laboratory tests were normal for Cases 7 and 8, for Case 6 , none were available. No amniotic fluids nor placental samples were taken from any of the three cases in this group. One mother (Case 6) reported clinical signs of ZIKV infection in early pregnancy, but no 
TABLE 2

Imaging findings in fetuses and newborns with congenital cerebral malformations and dysfunction following the 2013-2014 Zika virus outbreak, French Polynesia, 2014-2015 (n=19)

\begin{tabular}{|c|c|c|c|c|c|c|c|c|c|c|}
\hline \multicolumn{11}{|c|}{ Prenatal findings } \\
\hline & $\begin{array}{l}\text { Term at MRI, } \\
\text { WG + D }\end{array}$ & $\begin{array}{c}\text { Term at US scan, } \\
\text { WG + D }\end{array}$ & Gyration & VM (mm) & $\mathrm{OPC}$ & $\mathrm{ACC}$ & ASP & $\begin{array}{c}\text { Vermis } \\
\text { (percentile) }\end{array}$ & EPS & Calcifications \\
\hline \multicolumn{11}{|c|}{ Group 1a: Fetal brain abnormalities and microcephaly } \\
\hline Case 1 & $35+3$ & 34 & PMG & 13 & o & o & o & $13^{\circ}$ & + & + \\
\hline Case 2 & $28+4$ & $29+3$ & PMG/OD & 12 & + & 0 & o & $\mathrm{N}$ & + & + \\
\hline Case 3 & $30+1$ & $29+2$ & PMG/OD & 15 & + & + & + & $13^{\circ}$ & + & + \\
\hline Case 4 & 25 & 22 & PMG/OD & 10 & o & + & + & $\mathrm{N}$ & + & + \\
\hline Case 5 & 0 & 21 & NA & 10 & o & + & + & 0 & 0 & + \\
\hline \multicolumn{11}{|c|}{ Group 1b: Newborns with cerebral lesions and severe microcephaly } \\
\hline Case 6 & 0 & $34+2$ & NA & 10 & NA & NA & NA & $\mathrm{N}$ & NA & NA \\
\hline Case 7 & $32+4$ & $30+5$ & PMG/OD & 14 & + & + & + & $\mathrm{N}$ & + & + \\
\hline \multicolumn{11}{|c|}{ Group 2: Fetal brain abnormalities without microcephaly } \\
\hline Case 9 & 24 & $21+4$ & OD & 23 & 0 & 0 & Rupture & $\mathrm{N}$ & 0 & 0 \\
\hline Case 10 & 0 & 24 & NA & 16 & 0 & + & + & 0 & 0 & 0 \\
\hline Case 11 & $28+2$ & 28 & OD & 22 & o & 0 & Rupture & $\mathrm{N}$ & 0 & 0 \\
\hline Case 12 & 0 & $19+6$ & NA & 12 & 0 & 0 & o & $13^{\circ}$ & 0 & 0 \\
\hline Case 13 & 0 & $22+5$ & NA & 15 & 0 & + & + & $\mathrm{N}$ & 0 & 0 \\
\hline Case 14 & 0 & $24+5$ & NA & 17 & 0 & + & + & $\mathrm{N}$ & 0 & 0 \\
\hline \multicolumn{11}{|c|}{ Post-natal findings } \\
\hline & $\begin{array}{l}\text { Age at MRI } \\
\text { (month) }\end{array}$ & $\begin{array}{l}\text { Age at CT scan } \\
\text { (month) }\end{array}$ & Gyration & VM (mm) & OPC & $\mathrm{ACC}$ & ASP & $\begin{array}{c}\text { Vermis } \\
\text { (percentile) }\end{array}$ & ECM & Calcifications \\
\hline \multicolumn{11}{|c|}{ Group 1b: Newborns with cerebral lesions and severe microcephaly } \\
\hline Case 8 & 1 & 0 & PMG/OD & $\mathrm{N}$ & + & o & o & $\mathrm{N}$ & 0 & o \\
\hline \multicolumn{11}{|c|}{ Group 4: Newborns with congenital brainstem dysfunction } \\
\hline Case 15 & 1 & 19 & $\mathrm{~N}$ & $\mathrm{~N}$ & 0 & 0 & o & $\mathrm{N}$ & 0 & + \\
\hline Case 16 & 1 & NA & $\mathrm{N}$ & $\mathrm{N}$ & 0 & 0 & 0 & $\mathrm{~N}$ & + & 0 \\
\hline Case 17 & 1 & 14 & $\mathrm{~N}$ & $\mathrm{~N}$ & 0 & 0 & 0 & $\mathrm{~N}$ & + & + \\
\hline Case 18 & 0.3 & NA & $\mathrm{N}$ & $\mathrm{N}$ & 0 & 0 & 0 & $\mathrm{~N}$ & + & 0 \\
\hline Case19 & 1 & 8 & $\mathrm{~N}$ & $\mathrm{~N}$ & 0 & 0 & 0 & $\mathrm{~N}$ & 0 & 0 \\
\hline
\end{tabular}

ACC: absence of corpus callosum; ASP: absence of cavum septi pellucidi; D: day of gestation; ECM: enlarged cisterna magna; EPS: enlarged pericerebral space; MRI: magnetic resonance imaging; N: normal; NA: not available; OD: opercular dysplasia; OPC: occipital pseudo-cyst; PMG: polymicrogyria; o: none; +: present; VM: ventriculomegaly; WG: weeks of gestation.

serological test was performed. The two other mothers (Cases 7 and 8) were asymptomatic during pregnancy.

\section{Group 2}

For the six fetuses in this group, the head circumferences at birth were above the third percentile (range 5-90) or looked normal on pictures. However, all had at least two severe brain damages on imaging (Table 2): ventriculomegaly $\geq 12 \mathrm{~mm}(n=6)$, absence of the corpus callosum and cavum septi pellucidi $(n=3)$, opercular dysplasia $(n=2)$, and vermian agenesis $(n=2)$. These severe lesions led to TOP for all of them. Four of the six fetuses, additionally had extra-cerebral lesions: facial dysmorphia, laparoschisis and fetal akinesia with multiple pterygyum (Case 9); hydrops fetalis, fetal akinesia, rachischisis (Case 10); fetal akinesia (Case 11), and rachischisis (Case 12).

Three cases showed placental calcifications (Case 12, 13 and 14).
Viral tests were done on two amniotic fluids available (cases 12 and 13) and PCR was negative for ZIKV. Two mothers (Cases 11 and 14 ) reported symptoms of ZIKV infection in the first trimester (Figure 4).

\section{Group 3}

The five newborns in this group had clinical evidence of brainstem dysfunction, manifest by absence of sucking and swallowing, but without microcephaly or severe cerebral radiological anomalies. Prenatal US scan revealed in three cases polyhydramnios as consequence of early swallowing deficiency (Cases 17, $18,19)$. Head circumference was normal at birth for all cases. They were admitted to the neonatal ICU, due to severe feeding disorders, need for frequent aspiration and cardiac dysautonomia. Two cases (Cases 15 and 17) had an associated Pierre Robin Sequence (PRS) with retrognathia, glossoptosis and posterior U-shaped 
cleft palate, and a Moebius syndrome i.e. palsy of sixth and seventh cranial nerves. One of them (Case 17) required a tracheostomy and the other also had clubfeet and epilepsy (Case 15). One case had renal hypoplasia (Case 16). Cardiac dysautonomia manifested by attacks of bradycardy or tachycardy, was constantly present in all five cases.

All five were extensively investigated in Hôpital Necker, Paris, or Starship Hospital, Auckland, where most aetiologies for the brainstem dysfunction i.e. infectious, syndromic, genetic, metabolic were ruled out. Two cases (Cases 16 and 18) died in their first year of life at three (Case 16) and nine months (Case 18), respectively, likely due to parasympathetic cardiac dysregulation and respiratory distress. CGH array was normal for the three cases still alive. The two cases with PRS and Moebius syndrome (Cases 15 and 17) had periventricular and bulbar microcalcifications on CT-scans. Three cases had benign isolated enlarged cisterna magna on MRI. All five cases required gastrostomy tube feeding and frequent pharyngeal aspirations. Two (Cases 18 and 19) needed transient non-invasive positive pressure ventilation. The three living cases (Cases 15, 17, 19) are beginning to swallow and those with PRS have delayed neurological development.

No amniotic fluid was available for any of the five cases. Pregnancies were uneventful, besides urinary tract infection for one mother (Case 16). Mothers reported no ZIKV infection symptoms during pregnancy. In-house ELISA flavivirus serology revealed IgG in four of the five mothers. Two of them had neutralising antibodies for dengue serotypes 1-4, but no ZIKV neutralising antibodies. In the two others, no neutralising antibodies were detected for ZIKV, DEN, West Nile and Japanese encephalitis virus.

\section{Discussion}

Congenital cerebral malformations are rather rare in French Polynesia and are not collected in a register of congenital malformations. After 2011, when the prenatal diagnosis unit records started, about 15 congenital cerebral malformations were observed in 2012 and 2013, respectively and according to PMSI data, between 2001 and 2013, only two cases of brainstem dysfunction were noted in 2009 , leading to an average annual incidence rate of 0.34 cases per 10,000 births. Seven cases of congenital microcephaly were reported in the same period, equivalent to an average annual incidence rate of 1.2 cases per 10,000 live births. The present cluster of severe cerebral malformations with a 14 -fold increase in congenital microcephaly and 31-fold in brainstem dysfunction was spatially and temporally associated with a large outbreak of ZIKV in French Polynesia.

In Brazil, the detection of ZIKV genome in amniotic fluid [9-11] and in fetal brains of children with microcephaly $[10,12,13]$, concurrent with widespread local ZIKV transmission $[3,4,9]$ strengthened the hypothesis of teratogenicity of ZIKV. In our series, four fetuses who were infected in utero with ZIKV had typical symptoms of viral fetopathy, with microcephaly, severe brain lesions, intrauterine growth retardation and placental calcifications. Infectious virus was isolated from the amniotic fluid of four microcephaly cases, and clinical symptoms of ZIKV infection in the first trimester of pregnancy were reported by three of their mothers. However, ZIKV was not detected in the available amniotic fluids of three other cases tested (one microcephalic, two non-microcephalic), and their mothers were asymptomatic for ZIKV infection, as $80 \%$ of the population infected with ZIKV [2]. This finding raises the question about the possible correlation between maternal symptoms, probably associated with high viraemia, and the risk of transplacental transmission.

In the three microcephalic newborns in Group 1b, no further malformations were associated, besides micropenis. Another case of micropenis was found in Group 1a; a possible explanation for this finding could be central hormonal deficiency. Although there was no laboratory evidence for ZIKV infection in all three cases in Group $1 \mathrm{~b}$ and no ZIKV infection symptoms reported in two of three mothers, the severity of the microcephaly and of the radiologic brain lesions similar to those of group 1a, supports the hypotheses of probable ZIKV infection in the context of the ZIKV outbreak.

Of six cases with cerebral congenital malformations without microcephaly in Group 2, two mothers reported ZIKV infection symptoms in pregnancy. Unlike the other mothers of the same group, none of these mothers had fetuses with extracerebral malformation, besides fetal akinesia which could have been due to severe brain damages or peripheral neuropathy. One case with hydrops and brain lesions had not been explored for ZIKV infection. In Brazil, a fetus with hydrops, hydranencephaly and cranial calcifications was associated with a positive RT-PCR of ZIKV in brain and amniotic fluid [13].

The wide spectrum of clinical and neuroradiological findings in our microcephalic and non-microcephalic foetuses and newborns series suspected to be associated with ZIKV infection, may reflect the timing and severity of fetal viral infection. Early viral infection within the first trimester of pregnancy may interfere with neurocorticogenesis by impairing neuronal proliferation and migration between 12 and 24 WG, which leads to severe microcephaly [14] and neuronal cell migration disorders, whereas second semester infection may cause brainstem dysfunction without obvious lesions and intellectual disabilities. Microcephaly has been diagnosed as early as 21 WG in one fetus. By 25 to 30 WG, cortical gyrification can be analysed by $\mathrm{MRI}$ and in microcephalic fetuses, migration disorders were constantly present. The first signs of cerebral damage can be identified by the second trimester of pregnancy by US scan [15] when it reveals ventriculomegaly, destruction of the corpus callosum and/or of 
the cavum septi pellucidi and cerebral cysts. Occipital subependymal pseudocysts, which seemed to be another frequent hallmark visible on US scans in four of seven of our explored microcephalic cases, may suggest the consequences of the destruction of the germinative zone by the ZIKV, similar to what was observed in CMV infection [16].

Congenital brainstem dysfunction includes several symptoms such as sucking deficiency, aspiration, upper airways obstruction, and a possible PRS with a posterior and vertical position of the tongue [17]. It may be isolated or syndromic (CHARGE syndrome, etc.) [18]. Besides genetic causes, it could be due to an injury of the brainstem during embryonic or fetal life, such as an ischaemic stroke, misoprostol teratogenicity, maternal cocaine use or later viral infection [19] of the brainstem. The latter can be suspected for two of our cases with microcalcifications in the pons region. There was no evidence of ZIKV congenital infection, nor other proven aetiology, in any of our patients with congenital brainstem dysfunction (Group 3), and their mothers did not report any viral symptoms consistent with ZIKV infection during pregnancy.

The main limitations of our study are those of a retrospective review with bias of collecting data and loss of biological fluids which are stored for one year only. We have chosen to include only the severe brain malformations or dysfunction in our study. A surveillance artefact could result from missing data due to lack of an official register of congenital malformations, or due to non-exhaustive PMSI data and the risk that less severe cases who are not hospitalised were not notified which would have led to less cases having been noted. As concerns severe cases, the nature of the symptoms seems to make it unlikely that we have missed such cases while as pointed out above, milder cases could have been missed. Other postnatal lesions such as hearing, visual, cognitive impairments and epilepsy that could manifest later in life might be reported in further prospective studies in populations exposed to ZIKV.

\section{Conclusion}

ZIKV was for many years thought to be a benign febrile illness. In line with findings in Brazil, we retrospectively identified an unusual and heterogeneous cluster of congenital brain malformations and brainstem dysfunction in fetuses and newborns over a limited period following a ZIKV epidemic in French Polynesia. Except for four cases of fetal microcephaly, presenting with infectious virus in amniotic fluid and further confirming the Brazilian experience [9-13], we found no evidence of ZIKV vertical transmission for additional 15 cases; however, we were only able to perform ZIKV RT-PCR for three of them and we have no more opportunity to prove or to exclude the ZIKV infection in the 12 remaining cases. The spacio-temporal association might explain brain malformations in context of a viral outbreak. A case-control study is planned to look for other risk factors for these unexplained fetal and newborn abnormalities.

Given the potentially substantial public health implications of our findings, we strongly encourage researchers and clinicians to ensure that non-microcephalic congenital abnormalities, extra-cerebral malformations and brainstem dysfunction are included in their viral investigations for the potential teratogenicity of ZIKV.

\section{Acknowledgements}

We thank Eric Nilles and Sandrine Passemard for reviewing our work.

\section{Conflict of interest}

None declared.

Authors' contributions

MB and LM collected the data.

DEG, PGA and CG reviewed the imaging examinations.

SL, FR, ILG performed the viral investigations.

FBB reviewed the placenta samples.

VA managed the patients and helped with diagnostic and therapeutic care.

MLM and JMJ counselled for the prenatal management of fetuses.

HPM supervised the investigation, retrospective work and performed the epidemiological study.

$M B, D E G, V A, F R$ and HPM wrote the paper.

\section{References}

1. Ioos S, Mallet HP, Leparc Goffart I, Gauthier V, Cardoso T, Herida M. Current Zika virus epidemiology and recent epidemics. Med Mal Infect. 2014;44(7):302-7. DOI: 10.1016/j. medmal.2014.04.008 PMID: 25001879

2. Kucharski AJ, Eggo RM, Mallet HP, Edmunds J, Nilles EJ. Transmission dynamics of Zika virus in island populations: a modelling analysis of the 2013-2014 French Polynesia outbreak.bioRxiv. 2016;7. DOI: 10.1101/038588

3. European Centre for Disease Prevention and Control (ECDC). Rapid risk assessment: Microcephaly in Brazil potentially linked to the ZIKV epidemic. Nov 2015. Available from: http://ecdc.europa.eu/en/publications/Publications/zikamicrocephaly-Brazil-rapid-risk-assessment-Nov-2015.pdf

4. Pan American Health Organization (PAHO) / World Health Organization (WHO). Epidemiological alert. Increase in microcephaly in the northeast of Brazil. Washington DC: PAHO / WHO; 17 Nov 2015. Available from: http://www.paho.org/hq/ index.php?option=com_docman\&task=doc_view\&Itemid=270 \& gid $=32636 \&$ lang $=$ en

5. Besnard M, Mallet HP. Increase of cerebral congenital malformations among newborns and foetus in French Polynesia, 2014-2015, following a ZIKV outbreak. [Accessed 31 Mar 2016]. Available from: https://app.box.com/s/ ptvqdargwr10m3ag849rv59c5iditc07

6. Haute Autorité de Santé (HAS). Suivi et orientation des femmes enceintes en fonction des situations à risque identifiées. [Monitoring and guidance of pregnant women based on identified risk situations]. May 2007. Saint-Denis: HAS. French. Available from: http://www.has-sante.fr/portail/ 
$\mathrm{jcms} / \mathrm{c}_{2} 547976 / \mathrm{fr} / \mathrm{suivi-et-orientation-des-femmes-enceintes-}$ en-fonction-des-situations-a-risque-identifiees

7. World Health Organization (WHO). International Statistical Classification of Diseases and Related Health Problems 1oth Revision. ICD-10 Version: 2016. Geneva: WHO. Available from: http://apps.who.int/classifications/icd10/browse/2016/en

8. Association des Utilisateurs de Dossiers Informatisés en Pédiatrie. Obstétrique et Gynécologie (AUDIPOG). Head circumference charts. [Accessed 31 Mar 2016]. Available from: http://www.audipog.net/pdf/courbes_pc.pdf

9. Brazilian Medical Genetics Society-Zika Embryopathy Task Force,Schuler-Faccini L, Ribeiro EM, Feitosa IM, Horovitz DD, Cavalcanti DP, Pessoa A, et al. . Possible association between zikavirus infection and microcephaly, Brazil 2015.MMWR Morb Mortal Wkly Rep. 2016;65(3):59-62. DOI: 10.15585/mmwr. mm6503e2 PMID: 26820244

10. Martines RB, Bhatnagar J, Keating MK, Silva-Flannery L, Muehlenbachs A, Gary J, et al. Notes from the Field: Evidence of Zika Virus Infection in Brain and Placental Tissues from Two Congenitally Infected Newborns and Two Fetal Losses - Brazil, 2015. MMWR Morb Mortal Wkly Rep. 2016;65(6):159-60. DOI: 10.15585/mmwr.mm6506e1 PMID: 26890059

11. Oliveira Melo AS, Malinger G, Ximenes R, Szejnfeld PO, Alves Sampaio S, Bispo de Filippis AM. Zika virus intrauterine infection causes fetal brain abnormality and microcephaly: tip of the iceberg? Ultrasound Obstet Gynecol. 2016;47(1):6-7. DOI: 10.1002/uog.15831 PMID: 26731034

12. Mlakar J, Korva M, Tul N, Popović M, Poljšak-Prijatelj M, Mraz J, et al. ZIKV associated with microcephaly. N Engl J Med. 2016;374(10):951-8. DOI: 10.1056/NEJMoa1600651 PMID: 26862926

13. Sarno M, Sacramento GA, Khouri R, do Rosário MS, Costa F, Archanjo G, et al. Zika Virus Infection and Stillbirths: A Case of Hydrops Fetalis, Hydranencephaly and Fetal Demise. PLoS Negl Trop Dis. 2016;10(2):e0004517. DOI: 10.1371/journal. pntd.0004517 PMID: 26914330

14. Silasi M, Cardenas I, Kwon JY, Racicot K, Aldo P, Mor G. Viral infections during pregnancy.Am J Reprod Immunol. 2015;73(3):199-213. DOI: 10.1111/aji.12355 PMID: 25582523

15. Tarrant A, Garel C, Germanaud D, de Villemeur TB, Mignot C, Lenoir $M$, et al. Microcephaly: a radiological review. Pediatr Radiol. 2009;39(8):772-80, quiz 888-9. DOI: $10.1007 /$ s00247009-1266-x PMID: 19437006

16. Esteban H, Blondiaux E, Audureau E, Sileo C, Moutard ML, Gelot A, et al. Prenatal features of isolated subependymal pseudocysts associated with adverse pregnancy outcome. Ultrasound Obstet Gynecol. 2015;46(6):678-87. DOI: 10.1002/ uog.14820 PMID: 25684100

17. Abadie V, Morisseau-Durand MP, Beyler C, Manach Y, Couly G. Brainstem dysfunction: a possible neuroembryological pathogenesis of isolated Pierre Robin sequence. Eur J Pediatr. 2002;161(5):275-80. DOI: 10.1007/s00431-002-0936-6 PMID: 12012224

18. Abadie V, Couly G. Congenital feeding and swallowing disorders. Handb Clin Neurol. 2013;113:1539-49. DOI: 10.1016 B978-0-444-59565-2.00024-1 PMID: 23622377

19. Holder-Espinasse M, Abadie V, Cormier-Daire V, Beyler C, Manach Y, Munnich A, et al. Pierre Robin sequence: a series of 117 consecutive cases. J Pediatr. 2001;139(4):588-90. DOI: 10.1067/mpd.2001.117784 PMID: 11598609

\section{License and copyright}

This is an open-access article distributed under the terms of the Creative Commons Attribution (CC BY 4.0) Licence. You may share and adapt the material, but must give appropriate credit to the source, provide a link to the licence, and indicate if changes were made.

This article is copyright of the authors, 2016. 